\title{
Diacronie
}

Studi di Storia Contemporanea

$\mathrm{N}^{\circ} 31,3$ | 2017

“Le armi della politica, la politica delle armi"

\section{I diari di Enzo Ponzi}

L'esperienza di guerra in Croazia di un capitano dell'ufficio Propaganda (maggio-novembre 1942)

\section{Francesco Mantovani}

\section{Q OpenEdition}

\section{Journals}

\section{Edizione digitale}

URL: http://journals.openedition.org/diacronie/6171

DOI: $10.4000 /$ diacronie.6171

ISSN: 2038-0925

\section{Editore}

Association culturelle Diacronie

Notizia bibliografica digitale

Francesco Mantovani, «I diari di Enzo Ponzi », Diacronie [Online], №31, 3 | 2017, documento 3, Messo online il 29 octobre 2017, consultato il 04 mai 2019. URL : http://journals.openedition.org/ diacronie/6171; DOI : 10.4000/diacronie.6171 


\section{Diacronie}

Studi di Storia Contemporanea

$31,3 / 2017$

"Le armi della politica, la politica delle armi". Ideologie di lotta ed esperienze di guerra

\section{I diari di Enzo Ponzi. L'esperienza di guerra in Croazia di un capitano dell'ufficio Propaganda (maggio-novembre 1942)}

Francesco MANTOVANI

Per citare questo articolo:

MANTOVANI, Francesco, «I diari di Enzo Ponzi. L'esperienza di guerra in Croazia di un capitano dell'ufficio Propaganda (maggio-novembre 1942)», Diacronie. Studi di Storia Contemporanea : "Le armi della politica, la politica delle armi". Ideologie di lotta ed esperienze di guerra, 31, 3/2017, 29/10/2017,

URL: < http://www.studistorici.com/2017/10/29/mantovani_numero_31/ >

Diacronie Studi di Storia Contemporanea $\rightarrow$ http://www.diacronie.it Rivista storica online. Uscita trimestrale.

redazione.diacronie@hotmail.it

Comitato di direzione: Naor Ben-Yehoyada - João Fábio Bertonha - Christopher Denis-Delacour - Maximiliano Fuentes Codera Anders Granås Kjøstvedt - John Paul Newman - Deborah Paci - Niccolò Pianciola - Spyridon Ploumidis - Wilko Graf Von Hardenberg

Comitato di redazione: Jacopo Bassi - Luca Bufarale - Gianluca Canè - Fausto Pietrancosta - Alessandro Salvador - Matteo Tomasoni Diritti: gli articoli di Diacronie. Studi di Storia Contemporanea sono pubblicati sotto licenza Creative Commons 3.0. Possono essere

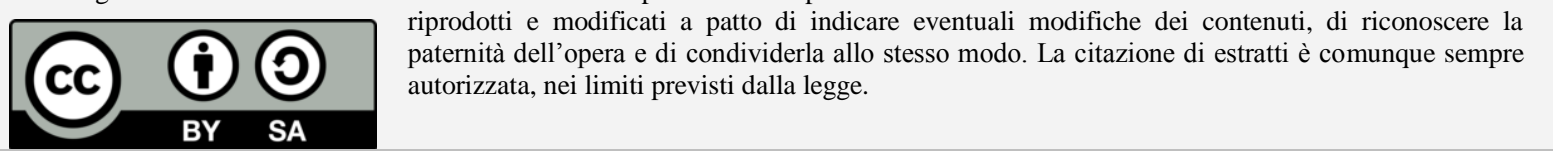




\section{2/ I diari di Enzo Ponzi. L'esperienza di guerra in Croazia di un capitano dell'ufficio Propaganda (maggio-novembre 1942)}

Francesco MANTOVANI

Nel panorama della rioccupazione dello Stato indipendente croato, in seguito ai massacri compiuti dagli ustascia nell'estate 1941, si colloca l'esperienza dell'avvocato modenese Enzo Ponzi in qualità di capo dell'ufficio Propaganda della Divisione Sassari, di stanza a Knin. I due diari del capitano Ponzi coprono maggio e ottobrenovembre 1942, mesi in cui la posizione delle truppe italiane si fece sempre più complicata, visto l'intensificarsi delle attività partigiane. Da queste pagine di memorialistica, accanto a notizie di carattere personale, emerge una panoramica sul «fronte interno» balcanico che va dai compiti presso l'ufficio Propaganda e dai rapporti con gli altri ufficiali di divisione, fino all 'impossibile alleanza in chiave anticomunista tra Regio Esercito, cetnici e ustascia.

Elenco delle abbreviazioni degli archivi citati

AISMO = Archivio dell' 'Istituto storico della Resistenza di Modena

AUSSME = Archivio dell'Ufficio storico dello Stato maggiore dell'Esercito

\section{Introduzione: Enzo Ponzi e il ritorno alle armi}

Molto più che i libri di storia $\mathrm{o}$ i documenti ufficiali, le fonti memorialistiche possiedono una capacità evocativa sorprendente. Al contrario però dei numerosi testi scritti nel dopoguerra da ex ufficiali o soldati, interessanti per indagare l'autopercezione a posteriori, i diari, redatti "in diretta", rappresentano una fonte di particolare interesse in quanto a essere riportate sono generalmente le impressioni immediate $o$, per meglio dire, mediate esclusivamente dalla visione di sé e del mondo contemporaneo ai fatti. Perciò, quello che interessa non sarà tanto la «verità effettuale della cosa», bensì come i

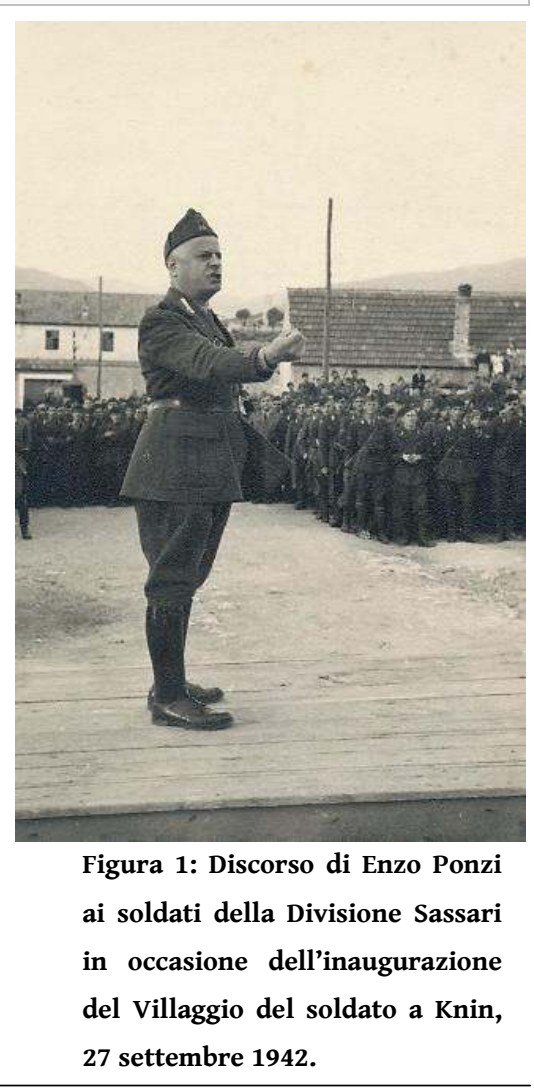

Figura 1: Discorso di Enzo Ponzi ai soldati della Divisione Sassari in occasione dell'inaugurazione del Villaggio del soldato a Knin, 
protagonisti di un determinato evento storico abbiano percepito il loro contemporaneo e la propria azione, proprio a partire da un orizzonte politico-culturale particolare.

Anche nello studio sull'occupazione italiana in Jugoslavia tra 1941 e 1943 da oltre vent'anni si è iniziato a utilizzare con padronanza di metodo alcune fonti memorialistiche, non più soltanto di alti gradi dell'esercito o politici di rilievo, ma anche di ufficiali inferiori, sottufficiali e soldati semplici, alcune delle quali sono state depositate presso l'Archivio diaristico nazionale di Pieve S. Stefano ${ }^{1}$. I due diari inediti del capitano Enzo Ponzi rappresentano pertanto uno strumento utile per lo studio dell'esperienza dell'occupazione italiana nei Balcani durante la seconda guerra mondiale ${ }^{2}$.

Personaggio di spicco del fascismo modenese, Enzo (Vincenzo) Ponzi nacque a Torino nel 1894. Trasferitosi a Modena per studiare legge, ad appena quattordici anni entrò nella redazione della «Gazzetta dell'Emilia», il più importante tra i giornali locali, col quale collaborò tra il 1912 e il 1915, fino cioè al suo arruolamento. Nel primo conflitto mondiale si distinse in diverse azioni militari, raggiungendo il grado di capitano della I Compagnia del $14^{\circ}$ Reparto d'Assalto e ottenendo alcune decorazioni ${ }^{3}$.

Tornato a Modena, aderì al primo Fascio locale, fondato il 27 maggio 1919 da ex combattenti, ufficiali, intellettuali e studenti. Si trattò però di un'esperienza breve, dal momento che nel settembre dello stesso anno egli, insieme a una decina di giovani fascisti, prese parte alla spedizione dannunziana di Fiume, privando così la sezione locale della propria base sociale. In seguito, il Fascio modenese venne rifondato il 16 novembre 1920 e a Enzo Ponzi venne affidata la carica di segretario. Vista l'esperienza giornalistica, egli diede vita, nell'aprile 1921, al periodico della federazione provinciale, «La Valanga». Tuttavia, la sua linea "moderata" finì presto per essere messa in minoranza dalla corrente più intransigente del Fascio di Modena, soprattutto dopo il patto di pacificazione stipulato a Roma col PSI il 2 aprile 1921: Ponzi, uno dei sostenitori di tale accordo, fu destituito e al suo posto fu nominato Carlo Zanni, rappresentante l'anima intransigente e movimentista del fascismo ${ }^{4}$.

\footnotetext{
${ }^{1}$ Per una bibliografia esaustiva, cfr. GOBETTI, Eric, «L'occupazione italiana in Jugoslavia (1941-1943). Storiografia e memoria pubblica», in Passato e presente, 30, 87, 3/2012, pp. 39-53.

${ }^{2}$ I due diari (3-19 maggio e 3 ottobre-18 novembre 1942) sono conservati presso l'Istituto storico di Modena, all'interno del Fondo Ponzi. Le fotografie e i documenti mostrati in questa sede sono stati allegati alle pagine dei diari Ponzi stesso.

${ }^{3}$ Nel novembre 1920 Ponzi querelò il settimanale socialista modenese «Il Domani», che lo accusava di aver ottenuto la medaglia di bronzo in seguito alla simulazione di un ferimento il 19 giugno 1918, a Fosso Palumbo, sulPiave. In seguito, a causa di "indelicatezze nel gioco", Ponzi venne punito con la degradazione da capitano a soldato semplice, fino addirittura all'espulsione dall'Associazione degli Arditi nel 1923. Solo nell'aprile 1939 ottenne di essere reintegrato nel grado di capitano, senza però riuscire a recuperare $\mathrm{i}$ ventidue anni di anzianità perduti. Cfr. ALBERGHI, Pietro, Modena nel periodo fascista (1919-1943), Modena, Mucchi-SIAS, 1998, p. 29n.

${ }^{4}$ Alberghi sottolinea come la "moderazione" di Ponzi non gli abbia impedito di rimanere per tutti gli anni successivi fascista, sebbene in contrasto con quelle frange della federazione modenese legate alla borghesia
} 
Rimasto tuttavia fervido collaboratore della federazione modenese, Ponzi tenne negli anni tra 1921 e 1924 un gran numero di conferenze, commemorazioni della vittoria della Grande guerra, inaugurazioni di gagliardetti e comizi elettorali in tutto il Nord Italia, soprattutto in EmiliaRomagna ${ }^{5}$. Nel settembre 1922 venne poi chiamato ad assumere la carica di segretario provinciale di Parma, per cercare di risolvere la difficile situazione sindacale del partito. Anche qui però, la politica di pacificazione andò presto a scontrarsi con la linea del sindacalismo contrario ad accordi con i "sovversivi" e, dopo qualche interruzione, Ponzi dovette dimettersi definitivamente dalla segreteria parmense all'inizio del $1924^{6}$. Nel febbraio dello stesso anno abbandonò il PNF, ma dopo pochi mesi decise di rientrarvi, seppur in posizione di secondo piano, e negli anni successivi si dedicò principalmente all'esercizio dell'avvocatura a Modena.

Enzo Ponzi tornò a impegnarsi direttamente in politica alla fine degli anni Trenta, riprendendo le attività di conferenziere e di giornalista che tanto lo avevano impegnato all'inizio del decennio precedente ${ }^{7}$. Ormai riabilitato, a metà aprile 1942 venne indicato dalla Federazione fascista di Modena come capo dell'ufficio «P» (Propaganda) presso il Comando d'Armata mobilitato, come vedremo nei prossimi paragrafi ${ }^{8}$. Tornato dalla guerra nel 1943, non aderì al Partito fascista repubblicano, soprattutto per la ferma opposizione della moglie, Elda Cristianini, cosa che gli permise di superare indenne la caduta della Repubblica di Salò e riprendere la carriera di avvocato fino alla sua morte, avvenuta il 17 giugno $1960^{9}$.

agraria e industriale, a cui Ponzi, in una lettera del 13 aprile 1921 al segretario generale del fascio, Umberto Passella, imputata le cause della sua destituzione. Cfr. Ibidem, pp. 16-17. In seguito, nel ripercorrere le vicende del 'biennio nero', Ponzi sottolineò come la violenza delle squadre fasciste fosse in realtà finalizzata alla restaurazione dello «Stato Nazionale», superando tutti i limiti di quello borghese. Cfr. PONZI, Enzo, Genesi e finalità del Fascismo, Modena, Premiata cooperativa tipografi, 1922, p. 19.

${ }^{5}$ Archivio dell'Istituto storico della Resistenza di Modena (d'ora innanzi AISMO), Fondo Enzo Ponzi, serie 1 “Carte personali 1804-1960", b. 156, fasc. 2 "Carteggio 1804-1960", I miei discorsi.

${ }^{6}$ Cfr. MINARDI, Marco, La nascita dei sindacati fascisti nel parmense (1921-1926), in DEGLI'INNOCENZI, Maurizio, POMBENI, Paolo, ROVERI, Alessandro (a cura di), Il Pnf in Emilia Romagna. Personale politico, quadri sindacali, cooperazione, Milano, Franco Angeli, 1988, pp. 239-255. Nello stesso periodo, Ponzi risulta essersi affiliato all'ordine di Salsomaggiore della massoneria, cfr. AISMO, Fondo Ponzi, b. 156, fasc. 2, Grande Oriente d'Italia, Gabinetto del Gran Maestro (27 dicembre 1922).

${ }^{7}$ Di questo ciclo di conferenze, a partire dall'inizio del 1940, è presente una vasta raccolta fotografica in AISMO, Fondo Ponzi, b. 157, fasc. 3 "Fotografie". A ciò si unisce l'attività di storico del fascismo modenese, portata avanti attraverso diverse pubblicazioni e quella, fino al maggio 1942, di curatore della rubrica Commentari di guerra per la "nuova" "Valanga», notiziario quindicinale dei Gruppi rionali del fascio di Modena.

${ }^{8}$ AISMO, Fondo Ponzi, b. 156, fasc. 1 “Diari, 1917-1944”, Diario 1942, 13 aprile 1942.

${ }^{9}$ Nonostante la mancata adesione ufficiale alla RSI, Ponzi e altri vecchi iscritti al PNF provarono in diverse occasioni ad avvicinarsi al partito repubblicano, finendo con l'esser considerati, come ebbe modo di scrivere nel suo diario, «traditori per i camerati e pur sempre odiati nemici per gli antifascisti». Cfr. AISMO, Fondo Ponzi, Diario 1944, 10 luglio 1944; FANTOZZI, Giovanni, Il volto del nemico. Fascisti e partigiani alla guerra civile (Modena 1943-1945), Modena, Artestampa, 2013, pp. 89-90. 


\section{Funerali e discorsi: l’ufficio «P» della Divisione Sassari}

Dopo essere stato ufficialmente richiamato in servizio dal Comando del Distretto Militare di Modena con un telegramma datato 3 maggio 1942, Enzo Ponzi viene assegnato alla sezione «P» della Divisone Sassari, dislocata nella Kninska Krajina, regione al confine tra lo Stato indipendente croato (Nezavisna Država Hrvatska, NDH), avente per capoluogo Knin, sede della Prefettura croata e del Comando di divisione italiano ${ }^{10}$.

La situazione della primavera 1942

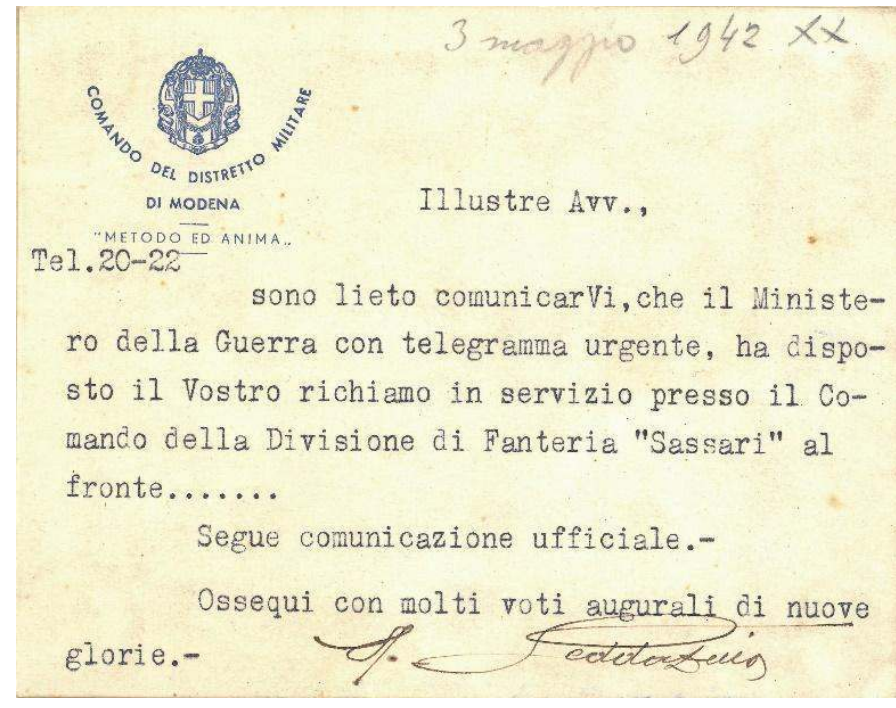

Figura 2: Lettera di richiamo in servizio dal Comando del Distretto di Modena per Enzo Ponzi, 3 maggio 1942.

non era delle più tranquille nelle zone occupate dagli italiani. Da un lato, la revisione delle competenze e dei compiti del Regio Esercito, ufficializzata dal bando del 7 settembre 1941, comportava l'assunzione dei poteri civili nella II zona d'occupazione (di cui faceva parte anche Knin) da parte del comandante della II Armata, il generale Vittorio Ambrosio: lo scopo era «pacificare» e «normalizzare» i rapporti tra croati e serbi, evitando il ripetersi dei massacri dell'estate compiuti dagli ustascia. D'altro lato, a causa dell'estendersi del movimento partigiano, alle questioni amministrative si aggiunsero quelle dalla repressione. Il successore di Ambrosio, il generale Mario Roatta, sulla base delle proprie esperienze coloniali decise il riordinamento di Supersloda (Comando Superiore Slovenia-Dalmazia, nome che assunse la II Armata dal maggio 1942) in grossi presidi. Se per certi versi questa riorganizzazione arrestò la ribellione in certe aree, soprattutto quelle urbane come Knin, tuttavia ciò contribuì alla formazione di zone «infestate» dai «ribelli» (secondo la terminologia ufficiale) ${ }^{11}$. Lo stesso Ponzi, durante il viaggio

\footnotetext{
${ }^{10}$ All'arrivo di Ponzi a Knin, la XXII Divisione Sassari - tredicimila uomini tra ufficiali e truppa - faceva parte del XVIII Corpo d'Armata (CdA) della II Armata. Nel maggio 1942 la Sassari era comandata dal generale Furio Monticelli, mentre il capo di Stato maggiore (SM) era il colonnello Gazzino Gazzini, redattore del diario storico della divisione. Archivio dell'Ufficio storico dello Stato maggiore dell'Esercito (d'ora in poi AUSSME), N 1-11, Diari storici, b. 523, Divisione Sassari.

${ }^{11}$ Per il bando del 7 settembre 1941 e l'arrivo di Roatta al comando di Supersloda, cfr. RODOGNO, Davide, Il nuovo ordine mediterraneo. Le politiche di occupazione dell'Italia fascista in Europa (1940-1943), Torino, Bollati Boringhieri, 2003, pp. 240-242; GOBETTI, Eric, L'occupazione allegra. Gli italiani in Jugoslavia (1941-1943), Roma, Carocci, 2007, pp. 83-84. Il generale Roatta è inoltre autore della «circolare 3 C» ( $1^{\circ}$ marzo 1942), in base alla quale «il trattamento da fare ai ribelli non deve essere sintetizzato dalla formula: "dente per dente" ma bensì da quella: "testa per dente"». Per il testo completo LEGNANI, Massimo (a cura di), «Il "ginger" del
} 
che lo portò a Knin, riporta di aver sentito che molti dei presidi più interni, rimasti isolati per lungo tempo durante l'inverno tra il 1941 e il 1942, abbiano subito diversi attacchi da parte delle formazioni partigiane e, per evitare ulteriori perdite, si avanzasse l'ipotesi di ritirarli dalla III zona d'occupazione, dove gli ustascia avevano avviato una nuova fase di rastrellamenti e persecuzioni ${ }^{12}$.

L'arrivo al capoluogo della Krajina, nel pomeriggio del 18 maggio, è quanto mai emblematico:

Appena metto piede a Knin sento la voce tremenda del cannone. Sono 24 anni che le mie orecchie non la sentivano e ne provo una certa emozione. [...] Bande di ribelli si sono spinte fino a $4 \mathrm{~km}$ da Knin, hanno aggredito e massacrato una colonna di truppa e altri reparti del $34^{\circ}$ Rgt. artigl. e del $151^{\circ}$ fant.: ci sono - sembra - almeno 48 morti. [...] Arrivo in un bel momento ${ }^{13}$.

Dopo essersi presentato al capo di SM, il colonnello Gazzini, Ponzi ottiene le prime istruzioni come capo dell'ufficio Propaganda: preparare i funerali per i caduti italiani del giorno, ventisette soldati e due ufficiali, a cui si aggiungono quarantasei cavalli uccisi, dodici feriti e un disperso. Alle 18 del giorno seguente, dopo aver preso accordi col cappellano militare e disposto i mezzi per il trasposto delle salme, si svolgono i funerali. Sarà questa una delle principali attività svolte dal capitano Ponzi nei mesi successivi, sia come organizzatore nel caso di esequie a soldati italiani, sia come rappresentante della divisione nel caso di truppe serbe o croate.

I sopralluoghi al cimitero di Knin, situato poco fuori dalla cittadina, costituivano a tutti gli effetti una sortita in territorio a rischio di imboscate, sempre più alto mano a mano che ci si avvicina alla fine del 1942. Per questa ragione, Ponzi riporta di essere accompagnato ogni volta da una scorta che andava dai quindici ai cinquanta soldati, generalmente armati di mitragliatrici e fucili mitragliatori, per proteggere i lavori di tumulazione dei caduti ${ }^{14}$. In altri casi, come per la commemorazione dei defunti del 2 novembre, fu necessario perfino modificare i piani della giornata: invece della cerimonia al cimitero, il generale Paolo Berardi, che da maggio 1942 aveva

generale Roatta. Le direttive della II Armata sulla repressione antipartigiana in Slovenia e Croazia», in Italia contemporanea, 209-210/1997-1998, pp. 155-174.

${ }^{12}$ AISMO, Fondo Ponzi, b. 156, fasc. 1 "Diari, 1917-1944", Diario, 17 maggio 1942. La voce giunta a Ponzi è confermata in GOBETTI, Eric, L'occupazione allegra, cit., pp. 96-97.

${ }^{13}$ AISMO, Fondo Ponzi, b. 156, fasc. 1 "Diari, 1917-1944", Diario, 18 maggio 1942. Si trattava di una batteria in trasferimento impiegata in un'azione congiunta con le bande četniche nella zona della valle di Plavno, contesa per tutto il mese di maggio con i partigiani. Cfr. TALPO, Oddone, Dalmazia. Una cronaca per la storia, vol. II, Roma, Stato maggiore dell'Esercito, 1990, p. 231.

${ }^{14}$ Durante il primo funerale, il 19 maggio, Ponzi dice di essere scortato da «una quindicina di mitraglieri»; qualche mese dopo, in occasione di una visita al cimitero per fare l'elenco degli italiani ivi sepolti, ad accompagnarlo sono «26 uomini e 1 ufficiale con fucili mitragliatori»; infine, nell'ultima pagina del secondo diario, per la sepoltura di un soldato della LXXIII legione Camicie nere (CC. NN.). la scorta ammonta invece a quaranta uomini. Cfr. AISMO, Fondo Ponzi, b. 156, fasc. 1 "Diari, 1917-1944", Diario, 19 maggio, 6 ottobre e 18 novembre 1942. 
sostituito Monticelli al comando della Sassari, aveva preferito fare celebrare una messa nella chiesa di Sv. Ante (Sant'Antonio) «per evitare - dice Ponzi - di esporre centinaia di ufficiali a un colpo di mano partigiano». Al termine della funzione, solo il generale, Ponzi e pochi ufficiali uscirono da Knin per posare una corona sul monumento ai caduti presso il cimitero, circondato da pattuglie di truppa, mentre «raffiche di mitragliatrici e di fucileria risuonano nella chiara mattinata soleggiata». Qui come in altri casi riportati nel diario, l'impressione che si ricava è quella di trovarsi in un perenne stato d'assedio, più o meno opprimente ${ }^{15}$.

L'arrivo del capitano Ponzi presso il comando della Sassari, nella primavera del 1942, al termine dell'operazione congiunta $\mathrm{Trio}^{16}$, si colloca proprio nel momento di passaggio da una prima fase, durante la quale la vita nei presidi italiani della II zona poteva dirsi relativamente tranquilla, a una seconda, in cui le caratteristiche della guerra nella penisola balcanica, dove era schierata circa la metà dell'intero esercito, mutarono profondamente. Quello che doveva essere il presidio di una nazione alleata, la Croazia, parte dello «spazio vitale» italiano, si tradusse prima in una gigantesca operazione di polizia, poi, con la nascita del movimento e dell'esercito partigiano, in una difficile guerra di logoramento che finì col compromettere il morale dell'esercito e col minare le premesse ideologiche di «missione civilizzatrice» che il regime fascista aveva dato all'occupazione dell'ex Jugoslavia. In questa cornice, lo Stato maggiore di ogni divisione si preoccupò di controllare e tenere alto l'umore delle truppe attraverso l'assistenza spirituale, lo sport e le attività sociali, la lettura, l'informazione e le case di tolleranza ${ }^{17}$.

Il diario di Enzo Ponzi offre un'importante testimonianza di come il nucleo «P» della Divisione Sassari gestisse buona parte di queste attività. Oltre all'organizzazione dei funerali, a Ponzi era assegnato il compito di tenere discorsi in varie occasioni, principalmente per celebrare ricorrenze e festività, come per il genetliaco di Vittorio Emanuele II e per il ventennale della marcia su Roma, o, d'altra parte, per commentare le notizie sull'andamento del conflitto. Nonostante il contenuto esatto di questi discorsi, tenuti generalmente verso le ore 18 presso il cinematografo di Knin, non venga quasi mai riportato per esteso, Ponzi annota pressoché ogni volta le reazioni della propria platea, soffermandosi in più occasioni sui commenti positivi espressi da ufficiali e truppa ${ }^{18}$.

\footnotetext{
${ }^{15}$ AISMO, Fondo Ponzi, b. 156, fasc. 1 “Diari, 1917-1944”, 2 novembre 1942.

${ }^{16}$ Per la cosiddetta operazione Trio (aprile-maggio 1942), che portò a un parziale successo nazi-fascista contro i partigiani in Bosnia, ma a un peggioramento delle situazioni per gli italiani in Erzegovina e Montenegro, cfr. BECHERELLI, Alberto, FORMICONI, Paolo, La quinta sponda. Una storia dell'occupazione italiana della Croazia. 1941-1943, Roma, Stato Maggiore della Difesa, 2015, pp. 66-73.

${ }^{17}$ Così RODOGNO, op. cit., pp. 183-185, 191-202. Anche i giornali di truppa, come «La Tradotta del Fronte Giulio» del Comando superiore di Supersloda e «Il Picchiasodo» dell' $11^{\circ}$ CdA, svolsero un ruolo importante di informazione e propaganda a partire dalla seconda metà del 1942 fino ai primi mesi dell'anno seguente. Cfr. SALA, Teodoro, «Guerriglia e controguerriglia in Jugoslavia nella propaganda per le truppe occupanti italiane (1941-1943)», in Il movimento di liberazione in Italia, 24, 108, 3/1972, pp. 91-114.

${ }^{18}$ In certi passaggi, traspare quasi un malcelato autocompiacimento per le reazioni positive, come quelle ricevute dopo il discorso fatto per il ventennale del fascismo, alla presenza di ufficiali italiani e autorità croate di Knin: «Parlo per 1 ora e $1 / 4$ sempre seguito con attenzione e interesse. Alla fine vengo applaudito,
} 
Uno dei pochi casi in cui è presente un breve riassunto di quanto abbia detto risulta essere il simposio organizzato il $1^{\circ}$ novembre per l'inaugurazione del corso di italiano, promosso dal PNF in accordo col Partito ustascia. Ponzi per prima cosa esprime con passione il proprio appoggio per tale iniziativa, segnale che, anche durante la guerra, l'uomo reclami i «diritti dello spirito», poi esalta la missione civilizzatrice di Roma: dice inoltre di aver ritrovato esposto presso un

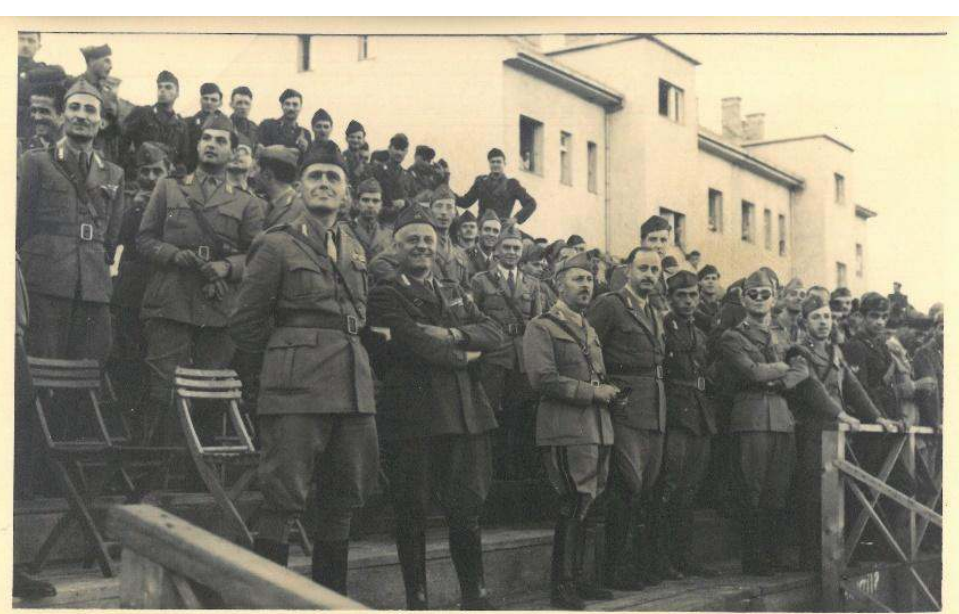

Figura 1: Torneo di calcio Divisione Sassari, Knin settembre-ottobre 1942. In primo piano da sinistra: gen. Berardi, cap. Ponzi, ten. col. Marabini, cap. Filippon. libraio croato una copia del De bello gallico di Cesare, quasi a conferma dell'eredità culturale lasciata dai latini i Croazia. L'altro punto su cui secondo quanto riportato - Ponzi insiste nel proprio intervento, è la proficua collaborazione tra Italia, Germania e NDH, non solo da un punto di vista militare, ma anche culturale, economico e commerciale. Come si vedrà più approfonditamente nel prossimo paragrafo, questo tipo di discorsi vanno ascritti ai tentativi compiuti dal regime e dall'esercito di non compromettere del tutto i rapporti tra italiani e croati, ormai instabili a causa dell'appoggio dato dall'esercito occupante alle formazioni cetniche ${ }^{19}$.

Come molte altre divisioni, anche la Sassari fu dotata di un «Villaggio del Soldato»: si trattava di sale di ritrovo dove i soldati potevano svagarsi con giochi da tavolo, carte e - nei casi più fortunati - con campi da bocce, pallacanestro e calcio. Nella seconda metà del 1942 vennero inaugurati a Knin il Villaggio del soldato (27 settembre) e, al suo interno, il «Circolo Ufficiali» (4 ottobre). Queste iniziative dei comandi militari e dei nuclei «P» avevano la doppia funzione di tenere impegnati i soldati attraverso le pratiche sportive, cercando di tenerne alto il morale, e di presentarsi alle autorità locali come potenza occupante ma fondamentalmente benevola, invitandole alle inaugurazioni e in altre occasioni importanti ${ }^{20}$.

anche il generale batte le mani, le autorità si felicitano e molti ufficiali mi esprimono la loro soddisfazione», cfr. AISMO, Fondo Ponzi, b. 156, fasc. 1 "Diari, 1917-1944", 28 ottobre 1942.

${ }^{19}$ Non è un caso che Ponzi dica di aver concluso il discorso inneggiando «al Duce, al Führer, al Poglavnik ["Guida", titolo di Ante Pavelić, NdA] e all'alleanza dei tre popoli». Cfr. AISMO, Fondo Ponzi, b. 156, fasc. 1 "Diari, 1917-1944", Diario, $1^{\circ}$ novembre 1942. Per la «missione civilizzatrice» ereditata dalla Roma antica, cfr. RODOGNO, op. cit., pp. 67-72.

${ }^{20}$ Il 4 ottobre, la domenica successiva all'apertura del villaggio del soldato, venne infatti organizzato un torneo di calcio tra l squadra degli artiglieri (Airone) e quella della fanteria (Rondine). Contestualmente, durante l'inaugurazione del circolo ufficiali, furono invitati anche autorità croate quali il viceprefetto Ante Vatavuk, il commissario distrettuale, e il vice-podestà di Knin Lace. Cfr. AISMO, Fondo Ponzi, b. 156, fasc. 1 "Diari, 1917-1944", Diario, 4 ottobre 1942 e passim per le fotografie. 
Tali edifici avevano d'altra parte un costo notevole, sia da un punto di vista di reperimento dei materiali sia per quanto riguarda i finanziamenti. Tra ottobre e novembre, quando il villaggio era già stato inaugurato, Ponzi sostiene che i lavori di completamento siano bloccati per mancanza di cemento: la ditta appaltatrice non ne ha, mentre il Genio militare «non può né vuole darne occorrendogli per lavori più urgenti e importanti», come quelli di fortificazione dei presidi. Qualche giorno dopo, il tenente colonnello del Genio Di Mattei dice a Ponzi che i costi dei lavori ammontano già a un milione di lire, senza per altro aver ottenuto il via libera del Comando del $\mathrm{CdA}^{21}$

D'altro canto, le ristrettezze materiali ed economiche non erano limitate ai grandi lavori. Come riportato da Rodogno, verso la fine del 1942 disciplina e il portamento disciplinato di ufficiali e truppa occupanti iniziarono infatti a scemare in comportamenti libertini, se non violenti o volti a speculazioni personali ${ }^{22}$. La sera del 2 novembre, facendo l'inventario dei doni portati dal federale di Spalato, Ferruccio Cappi, in visita alla Sassari, Ponzi constata «con dolore e umiliazione» che durante l'imballaggio o il viaggio le casse siano state manomesse e rubate, tra le altre cose, quattromila sigarette sulle quarantamila donate e nove bottiglie di liquori su cento. In un'altra pagina del diario, Ponzi lamenta di aver dovuto riempire il proprio ufficio con le casse delle cartoline con franchigia militare, per evitare che altri «lazzaroni» le svaligiassero ulteriormente, nonostante esse fossero distribuite alla truppa. Questi episodi, più che la «maledetta peste italiana del furto e dell'indisciplina» stigmatizzata da Ponzi, sembrano attestare l'esistenza di un mercato nero piuttosto diffuso, soprattutto per alcune categorie di merci (alcolici, sigarette e valori bollati), anche all'interno dei presidi militari ${ }^{23}$.

Malessere, ristrettezze e - dalla seconda metà del 1942 - i primi veri insuccessi militari dell'Asse contribuirono inoltre ad aumentare il sentimento di sfiducia verso il fascismo, anche se, fino all'estate del 1943, non si può parlare di un antifascismo consapevole. Se tra i soldati la disillusione si esprimeva generalmente attraverso comportamenti indisciplinati, anche alcuni ufficiali cominciarono a manifestare sfiducia verso il regime e, nello specifico, verso alcuni gerarchi ${ }^{24}$. Nel diario del capitano dell'ufficio Propaganda, attento a questo genere di comportamenti, sono presenti in vari punti critiche allo stesso generale Berardi e agli altri ufficiali del Comando di divisione, a volte per inconcludenza, altre invece per atteggiamenti che Ponzi non esita definire «antifascisti». Nei primi giorni di novembre le notizie della disfatta di el-

\footnotetext{
${ }^{21}$ Cfr. AISMO, Fondo Ponzi, b. 156, fasc. 1 “Diari, 1917-1944”, Diario, 21 ottobre e 9 novembre 1942.

${ }^{22}$ Per l'interiorizzazione della violenza, l'adattamento alla brutalità e i comportamenti del conquistatore «straccione e libertino», che comprendevano il concubinaggio con donne locali, i furti e l'alcolismo, cfr. RODOGNO, Davide, op. cit., 212-214 e 218-221.

${ }^{23}$ Per il furto di sigarette e alcolici, di cui Ponzi fece poi rapporto al federale di Spalato, cfr. AISMO, Fondo Ponzi, b. 156, fasc. 1 "Diari, 1917-1944", Diario, 2 e 3 novembre 1942; per quello delle cartoline con franchigia, cfr. Ibidem, 7 novembre 1942.

${ }^{24}$ Cfr. GOBETTI, Eric, L'occupazione allegra, cit., pp. 187-188.
} 
Alamein, degli sbarchi alleati in Marocco e Algeria e della conseguente occupazione della repubblica di Vichy da parte di Germania e Italia suscitarono tra alcuni ufficiali le "solite reazioni»: «non dolore, non smarrimento, non ansia ma quasi un senso di maligna soddisfazione di antifascisti che non temono di vedere rovinata la Patria purché sia rovinato il Partito» ${ }^{25}$. Neanche l'occupazione di Corsica e Nizza dell'11-12 novembre sembrò accendere negli ufficiali speranze per la loro annessione, cosa che invece Ponzi riteneva ormai scontata: al suo entusiasmo, il generale Berardi e altri ufficiali rispondono con una «deprimente» scrollata di spalle oppure come viene riportato - con un laconico «Ah sì??» ${ }^{26}$.

Nonostante quindi un grado di fascistizzazione del Regio Esercito tutt'altro che trascurabile, la maggior parte degli studi sullo scacchiere balcanico hanno dimostrato come, a causa dello smarrimento a cui ufficiali e truppa andarono incontro di fronte all'insurrezione partigiana e all'atteggiamento ostile delle popolazioni occupate, il tentativo fascista di ideologizzare il conflitto fosse fallito, perché i soldati non arrivarono a identificarsi con il modello imposto dal regime. Ciò tuttavia non comportò una minore durezza nell'eseguire le direttive dei piani di «normalizzazione» anche da parte di ufficiali non vicini al fascismo, tra gli altri lo stesso generale Ambrosio e il generale Mario Robotti27.

\footnotetext{
${ }^{25}$ Cit. in AISMO, Fondo Ponzi, b. 156, fasc. 1 "Diari, 1917-1944", Diario, 14 novembre 1942. Per l'occupazione di italiani e tedeschi dei territori liberi francesi e la scarsa autonomia che fu lasciata di fatto al Regio Esercito, cfr. RODOGNO, Davide, op. cit., pp. 57-59.

${ }^{26}$ Allo scetticismo di parte dei superiori, Ponzi contrappone invece l'entusiasmo dei seicento soldati che parteciparono alla sua conferenza sulla «liberazione» di Nizza e Corsica; poche pagine prima, egli addirittura elenca gli ufficiali che, durante la discussione alla mensa sull'andamento della guerra, si erano mostrati più o meno «scettici e antifascisti». Cfr. AISMO, Fondo Ponzi, b. 156, fasc. 1 "Diari, 1917-1944", Diario, 12 novembre 1942. Per quanto riguarda il gen. Berardi, il suo progressivo allontanamento dal regime fascista è confermato dalla sua nomina, dopo l'8 settembre 1943, a capo di SM dell'esercito del regno del Sud per affiancare le operazioni dell'VIII Armata britannica nelle operazioni sul fronte adriatico; Berardi figura poi tra gli ufficiali «discriminati» dalla Commissione d'inchiesta italiana per i crimini di guerra in Jugoslavia nel 1947. Cfr. NITTI, Gian Paolo, «Berardi, Paolo», in Dizionario biografico degli italiani, URL: <http://www.treccani.it/enciclopedia/paolo-berardi_(Dizionario-Biografico)/ > [consultato il 25 febbraio 2017]; «Nominativi sottoposti alla commissione d'inchiesta per i presunti criminali di guerra italiani (situazione al 25 gennaio 1947)», in Crimini di guerra, URL: <http://www.criminidiguerra.it/3ListeCriminali.shtml > [consultato il 15 febbraio 2017].

${ }^{27}$ Per limitata lunghezza del saggio, si rimanda all'ottima sintesi di GIUSTI, Maria Teresa, La Iugoslavia tra guerriglia e repressione: la memoria storiografica e le nuove fonti, in CACCAMO, Francesco, MONZALI, Luciano (a cura di), L'occupazione italiana della Jugoslavia (1941-1943), Firenze, Le Lettere, 2008, pp. 379-418. Per la politica di repressione portata avanti nella provincia di Lubiana e in Montenegro si segnalano i recenti studi: OSTI GUERRAZZI, Amedeo, The Italian Army in Slovenia Strategies of Antipartisan Repression, 1941-1943, New York, Palgrave Macmillan, 2013; GODDI, Federico, Fronte Montenegro. Occupazione italiana e giustizia militare (19411943), Gorizia, Libreria Editrice Goriziana, 2016. Non mancarono certo i casi di violenza prettamente ideologica ai danni della popolazione civile, soprattutto da parte delle CC. NN. La tesi sostenuta da studiosi come Collotti e Rodogno è però quella di una violenta repressione caratterizzata non tanto dal senso di superiorità razziale (come invece avvenne nel caso dei tedeschi), bensì come segno di debolezza e come unico modo per mostrarsi come «potenza occupante». Cfr. COLLOTTI, Enzo, Sulla politica di repressione nei Balcani, in PAGGI, Luca (a cura di), La memoria del nazismo nell'Europa di oggi, Firenze, La Nuova Italia, 1997, pp. 182-208; RODOGNO, Davide, op. cit., pp. 225-227.
} 


\section{Il piede in due staffe: italiani, ustascia e cetnici a Knin}

Fu proprio il rapporto con le popolazioni dello Stato indipendente croato a far venire a galla le debolezze della strategia italiana nel controllo del territorio: di ciò Enzo Ponzi è pienamente conscio e in numerosi passi del diario esprime il proprio scetticismo verso le politiche del Regio Esercito. Le relazioni italo-croate furono infatti caratterizzate da forti tensioni a vari livelli, dovute principalmente alla questione dalmata. Mentre i vertici militari italiani della II Armata (Ambrosio in primis) si mostreranno fin dall'inizio critici verso il governo ustaša, la Legazione a Zagabria, soprattutto l'incaricato d'affari italiano, Raffaele Casertano, porterà avanti fino a buona parte del 1943 una politica decisamente più conciliante, non esitando in diverse occasioni a criticare gli atteggiamenti filo-serbi di ufficiali e soldati italiani ${ }^{28}$.

Tale questione era di particolare importanza in una regione, la Krajina, nella quale la popolazione era alquanto eterogenea, con campagne prevalentemente ortodosse e alcuni centri ad alta percentuale cattolica. Di qui lo scarso successo che il movimento ustascia era riuscito a riscuotere prima del 1941 in questa zona ${ }^{29}$. Come si è visto nel paragrafo precedente, il bando del 7 settembre 1941 aveva cercato di «pacificare» le zone del NDH dove più cruenti erano stati i massacri da parte croata: tolta la libertà d'azione alla milizia ustascia, ai domobrani (i soldati dell'esercito regolare) e alla gendarmeria croata era stata lasciata la possibilità, in collaborazione coi carabinieri, di essere impiegati nei servizi di pubblica sicurezza30.

Nel corso del 1942, la convivenza tra le truppe della Divisione Sassari e la popolazione civile, la possibilità di mantenere l'ordine e la lotta contro il movimento partigiano divennero sempre più dipendenti dai buoni rapporti con le milizie cetniche e con i loro capi ${ }^{31}$. Dopo l'iniziale reticenza da parte dei comandi italiani nel servirsi delle formazioni nazionaliste serbe, soprattutto per paura di compromettere i rapporti con l'alleato croato, alcune di esse vennero impiegate in una serie di cicli operativi, tra marzo e giugno 1942, volti ad arginare il diffondersi della resistenza

\footnotetext{
${ }^{28}$ Per la ricostruzione dei contrasti tra diplomazia ed esercito sull'atteggiamento verso il governo di Zagabria, fondamentali sono le ricerche nell'Archivio Storico Diplomatico del Ministero degli Affari Esteri e nell'AUSSME. Cfr. MONZALI, Luciano, La difficile alleanza con la Croazia ustascia, in CACCAMO, Francesco, MONZALI, Luciano, op. cit., pp. 61-131, qui pp. 93-94; BECHERELLI, Alberto, Italia e Stato Indipendente Croato (1941-1943), Roma, Nuova Cultura, 2012, pp. 149-159; BECHERELLI, Alberto, FORMICONI, Paolo (a cura di), op. cit., pp. 106-107.

${ }^{29}$ Per riferimenti alla complessa questione delle «identità nazionali» serbe e croate e alla conformazione della Kninska Krajina, cfr. GOBETTI, Eric, L'occupazione allegra, cit., pp. 26 et seq.

${ }^{30}$ Proprio in una di queste azioni, condotte con pattuglie di carabinieri e truppe italiane da un lato e gendarmi e domobrani dall'altro, fu coinvolto Ponzi la sera del suo arrivo, in una Knin «deserta e illuminata dopo il coprifuoco». La prima impressione che il capitano dell'ufficio «P» ebbe degli ufficiali alleati, definiti "gente che val poco militarmente», non sembra essere stata molto positiva. Cfr. AISMO, Fondo Ponzi, b. 156, fasc. 1 “Diari, 1917-1944", Diario, 18 maggio 1942.

${ }^{31}$ Un primo accordo tra comando della Sassari e capi serbi risaliva in realtà alla fine di luglio 1941, durante la sollevazione contro i massacri compiuti dagli ustascia: anche in questo caso, l'impegno era di collaborare alla «pacificazione» della Krajina e alla lotta antipartigiana, cfr. BECHERELLI, Alberto, op. cit., pp. 135-136.
} 
comunista. La svolta nei rapporti italo-cetnici avvenne però solo in seguito ai massacri di giugno compiuti dagli ustascia contro le popolazioni serbe della III zona, sgomberata dalle truppe italiane. Viste le nuove violenze e l'inefficienza dell'esercito del NDH, Roatta promosse l'inquadramento delle bande četniche nella Milizia volontaria anticomunista (MVAC), in seguito agli accordi presi da una parte con Pavelić e dall'altra con i principali capi serbi. Poste sotto la supervisione del comandante italiano competente per territorio e di due ufficiali di collegamento, a queste formazioni furono affidati i compiti di difesa dei villaggi e dei centri abitati e di partecipazione ai cicli operativi del Regio Esercito32.

Figura di rilievo già prima della seconda guerra mondiale, il pope ortodosso Momčilo Đujić riuscì a imporsi come principale leader četnico nella zona di Knin, anche attraverso l'emarginazione e l'uccisione dei suoi principali concorrenti. La sua politica fortemente anticroata non gli impedì, il 19 giugno 1942, di sottoscrivere insieme ad altri notabili serbi un accordo col prefetto di Knin, David Sinčić, alla presenza delle autorità militari italiane, con cui si impegnavano nella comune lotta antipartigiana33.

Dei controversi rapporti tra italiani, croati e serbi sono presenti molte tracce nel diario di Ponzi, il quale si mostra generalmente critico sulla reale efficacia della MVAC, soprattutto perché l'aperto appoggio alle bande cetniche andava a compromettere i rapporti con Zagabria. Ai primi di ottobre 1942, durante un pranzo al Comando di divisione seguito al funerale di un miliziano cetnico, il capitano Ponzi descrive il discorso «molto prolisso» del pope Đujić, il quale ricorda le stragi compiute dagli ustascia sulle popolazioni serbe, di fronte alle quali soldati e ufficiali inferiori italiani mostravano compassione, mentre quelli superiori «lasciavano fare», seguendo le direttive di Roma. Di fronte quelle che sono vere e proprie accuse, il generale Berardi - dice Ponzi - non risponde e cerca anzi di «mandarli via contenti». Questo atteggiamento accondiscendente, unito al fatto che al funerale avesse presenziato un intero battaglione italiano, secondo Ponzi non potevano non essere percepita come

una leva in massa, una provocazione pei croati. E siamo nello stato croato alleato e amico, e armiamo i nemici dei croati, e ci alleviamo in seno il serpe. E non più tardi di iersera il

\footnotetext{
${ }^{32}$ Per l'accordo italo-croato del 19 giugno 1942, cfr. MONZALI, Luciani, op. cit., pp. 102-108; per l'evolversi degli ambigui rapporti italo-cetnici e la formazione della MVAC, che, secondo le cifre più attendibili, nell'estate 1942 contava nello Stato indipendente croato circa 12.000 uomini, cfr. TOMASEVICH, Jozo, War and Revolution in Yugoslavia, 1941-1945. The Chetniks, Stanford, Stanford University Press, 1975; RODOGNO, op. cit., pp. 376; FABEI, Stefano, I cetnici nella Seconda guerra mondiale. Dalla Resistenza alla collaborazione con l'esercito italiano, Gorizia, Libreria Editrice Goriziana, 2006; GOBETTI, L'occupazione allegra, op. cit., 108-114; CUZZI, Marco, «La strategia dell'ambiguità: i cetnici di Draža Mihailović», in Qualestoria, 43, 2/2015, pp. 3363.

${ }_{33}$ Per le controverse figure del pope-condottiero Đujić e del prefetto Sinčić, entrambe criticate rispettivamente dagli altri capi cetnici e dalle autorità dell'NDH, cfr. GOBETTI, L'occupazione allegra, op. cit., pp. 132-135; BECHERELLI, Alberto, op. cit., pp. 208, 321.
} 
generale riceveva le maggiori autorità croate e rivolgeva loro espressioni cordiali e amichevoli! Politica pericolosa quella del piede in due staffe 34 .

La tanto criticata arrendevolezza dei comandi italiani nei confronti soprattutto dei cetnici è, d'altro canto, sintomatica della difficoltà di mantenere l'ordine in una realtà altamente conflittuale, dove il richiamo alla lotta anticomunista non riusciva a sopire le rivalità esplose dopo il crollo della Jugoslavia35. Fu proprio il coinvolgimento nelle operazioni congiunte del cosiddetto ciclo Dinara, condotte tra ottobre e novembre nella Kninska Krajina e in Erzegovina per contenere l'avanzata partigiana, a essere l'occasione per riaprire le tensioni tra croati, cetnici e - di conseguenza - comandi italiani. Nel corso dell'operazione Alfa, prima parte questo ciclo operativo, nella quale il contributo della MVAC fu particolarmente rilevante (3.000-4.000 unità), i cetnici compirono una serie di saccheggi e uccisioni, sparando dai finestrini dei treni durante i trasferimenti e mettendo a ferro e fuoco alcuni villaggi delle zone a maggioranza cattolica, scatenando l'indignazione delle autorità centrali croate ${ }^{36}$.

Il fermento doveva essere ben percepito anche dai militari italiani che non prendevano parte direttamente alle operazioni. Il 5 ottobre Ponzi riporta la voce di un massacro di 800 persone compito da un battaglione della MVAC nei pressi di Spalato: «entrando nei paesi i cetnici facevano il saluto a pugno chiuso: la gente rispondeva con evviva al comunismo e allora si ammazzava». Al di là della cifra più o meno attendibile - egli stesso nutre qualche perplessità - secondo Ponzi si tratterebbe di un'ulteriore dimostrazione dell'«anarchia che qui regna e della contraddittorietà» della politica italiana nei confronti dei croati e dei serbi» 37.

Lo scetticismo sfociò in certi casi anche nel sospetto di tradimento. Nel corso degli attacchi dei partigiani al presidio italiano di Bosansko Grahovo, a Nord di Knin e quasi al confine tra II e III zona, secondo Ponzi diversi reparti cetnici si sarebbero «disorganizzati», passando in certi casi dalla parte dei ribelli ${ }^{38}$. Sebbene infatti la seconda fase dell'operazione Dinara, denominata Beta, avesse portato alla rapida avanzata italo-croata e alla riconquista di Livno il 23 ottobre, i partigiani non si limitavano più a ritirarsi, bensì «dimostravano una visione quasi professionale del campo di battaglia, con nuovi criteri tattici»39, attaccando le retrovie e i villaggi tenuti dai

\footnotetext{
${ }^{34}$ AISMO, Fondo Ponzi, b. 156, fasc. 1 “Diari, 1917-1944”, Diario, 5 ottobre 1942.

${ }^{35} \mathrm{Nel}$ corso di una riunione plenaria degli ufficiali per salutare la partenza del col. Gazzini, presso il circolo ufficiali, «serbi e croati si guardano un po' in cagnesco. Il colonnello se la cava chiamando nobili l'una e l'altra nazione, facendo l'elogio dei croati e dei serbi e inneggiando alla collaborazione anticomunista». Cfr. AISMO, Fondo Ponzi, b. 156, fasc. 1 “Diari, 1917-1944”, Diario, 9 ottobre 1942.

${ }^{36}$ Le vittime di queste violenze, secondo le fonti riportate da Gobetti, vanno dalle 500 alle 1.000 persone. Cfr. GOBETTI, Eric, L'occupazione allegra, cit., pp. 137-138; per l'operazione Alfa, cfr. TALPO, Oddone, op. cit., pp. 748-754

${ }^{37}$ AISMO, Fondo Ponzi, b. 156, fasc. 1 “Diari, 1917-1944”, Diario, 5 ottobre 1942.

${ }^{38}$ AISMO, Fondo Ponzi, b. 156, fasc. 1 “Diari, 1917-1944”, Diario, 27 ottobre 1942.

${ }^{39}$ TALPO, Oddone, op. cit., p. 761
} 
četnici, costringendoli a ritirarsi nei presidi, arrivando a minacciare le città durante la notte. Lo stesso Đujić il 27 ottobre fu costretto a lasciare Strmica, sede del quartier generale della divisione Dinara della MVAC, mentre pochi giorni dopo un suo parente rimase ucciso nel corso di un attacco al villaggio di Topolje ${ }^{40}$. Anche a Knin i partigiani riuscirono a compiere azioni dimostrative: la notte del 7 novembre comparve sul muro del villaggio del soldato una scritta che inneggiava al comunismo ${ }^{41}$.

Nonostante la pericolosa avanzata dei «ribelli», le tensioni tra ustaša e četnici non sembravano sopirsi. Una delle pagine più interessanti del diario di Ponzi è proprio la descrizione di un fatto da lui definito "abbastanza increscioso e allarmante», avvenuto sul campo sportivo di Knin il 4 novembre, nel corso della rivista del battaglione di domobrani del maggiore Tomislav Draganić, di quello del tenente colonnello Giovannino Biddau del $152^{\circ}$ reggimento di fanteria e delle «bande irregolari cetniche» comandate dall'ex ufficiale jugoslavo Bosko Asanović42. Dopo un breve discorso col quale il generale Berardi elogiò lo spirito di collaborazione anticomunista, davanti alla tribuna su cui si trovava insieme a Ponzi, ad altri ufficiali italiani e al maggiore croato sfilano per prime le truppe dell'NDH, cui fanno seguito quelle italiane. Arrivato il loro turno, i četnici non accennano a volersi muovere: il comandante serbo si avvicina allora alla tribuna e, stando sull'attenti, dice a Berardi di non aver intenzione di sfilare dopo i croati 43 .

Il magg. Draganic [sic] scende uno scalino, si avvicina al generale e dice: Noi ce ne andiamo. Il generale ribatte: Se volete compiere un'opera di civiltà rimanete. $\mathrm{E}$ fa ripetere al serbo che essi debbono sfilare e sfilando dinanzi a un generale italiano non si umiliano a nessuno. [...] Un altro capo si avvicina e rincalza le ragioni di Assanovic [sic]. Comunque finiscono per cedere e accettano di sfilare in senso avverso. Questa non l'ho ben capita. Certo si è che queste bande, zingaresche e pittoresche, vestite nelle più strane fogge, taluni con barbe e capelli alla nazzarena, con cappotti e mantelli e coperte borghesi, e salmerie, muli, asini, biciclette, armi di vari tipi, bombe alla cintola, sfilano anch'esse, e non si può loro disconoscere una certa prestanza militare 44.

\footnotetext{
${ }^{40}$ Sebbene le fonti italiane parlino di un «fratello» del pope, è possibile che si sia trattato di un cugino, visto che il termine brat in serbo-croato significa sia fratello che cugino. Cfr. AISMO, Fondo Ponzi, b. 156, fasc. 1 "Diari, 1917-1944", Diario, 27 ottobre e 12 novembre 1942; GOBETTI, Eric, L'occupazione allegra, cit., pp. 139140.

${ }^{41}$ La scritta era «Živio Staljin Živio Rusija», evviva Stalin, evviva la Russia. Cfr. AISMO, Fondo Ponzi, b. 156, fasc. 1 "Diari, 1917-1944", Diario, 7 novembre 1942.

${ }^{42} \mathrm{Per}$ il fatto e le citazioni che seguono, cfr. Ibidem, 4 nov. 1942. Le sottolineature sono nell'originale.

${ }^{43}$ Come in molte altre occasioni, la conversazione avviene nelle tre lingue dei militari presenti (serbo, croato e italiano): di qui l'importanza degli ufficiali mediatori, addetti anche alla traduzione. Nel caso del 4 novembre si tratta del capitano Vincenzo Marussi di Zara, dell'ufficio Informazioni della Sassari.

${ }^{44}$ A rivista finita, Ponzi racconta che, sebbene fossero stati smobilitati, gruppi di četnici continuino a girare per Knin fino a tarda notte, intonando canzoni anticroate.
} 
Oltre che per la suggestiva descrizione del vestiario dei četnici, il passo è di particolare rilevanza perché mostra l'instabilità della struttura di mediazione ideata dal generale Roatta, nella quale gli italiani avrebbero dovuto giocare un ruolo guida tra croati e serbi. L'episodio destò un certo imbarazzo al Comando di divisione della Sassari e si cercò in tutti i modi di non farlo trapelare 45 .

Nonostante i numerosi accordi firmati nel corso del 1942, la diffidenza reciproca permase e si manifestò spesso - come in questo caso - in questioni legate alla simbologia nazionale o al codice di onore militare. Le perplessità di Enzo Ponzi sulle formazioni serbo-nazionaliste erano d'altronde condivise da vaste componenti dei comandi italiani: i contatti presenti tra četnici e Alleati, il non completo inquadramento nella Milizia volontaria anticomunista e - soprattutto - il timore di compromettere definitivamente i rapporti con Zagabria spinsero gli alti comandi a limitare il più possibile gli aiuti ai nazionalisti serbi, sebbene proprio nel territorio della Sassari la MVAC avesse giocato un ruolo importante nelle operazioni di antiguerriglia ${ }^{46}$. Va comunque notato che l'ostilità dimostrata dai croati nei confronti di questa collaborazione non abbia impedito alle autorità di Zagabria di dialogare con alcuni capi delle bande cetniche, proprio per evitarne un il consolidamento dei rapporti tra Regio Esercito e serbi47.

La politica del «piede in due staffe» mise pertanto l'esercito d'occupazione in una posizione scomoda in primo luogo nei confronti dell'NDH, che dal febbraio 1943, quando Roatta e gli altri comandanti militari italiani rinunciarono al controllo politico-economico della II zona, entrò definitivamente a far parte della sfera d'influenza della Germania che, presentandosi come potenza priva di rivendicazioni territoriali nell'area, riuscì a guadagnarsi la fiducia del governo di Zagabria ${ }^{48}$. L'incrinarsi delle relazioni italo-croate andò a sommarsi, in secondo luogo, all'ordine da parte dei vertici tedeschi, alla fine del 1942, di smobilitare e disarmare i serbi nazionalisti, facendo così perdere la «carta cetnica» che gli italiani avevano sperato di poter giocare per mantenere il controllo dell'ex Jugoslavia49.

\footnotetext{
${ }^{45}$ Ponzi sostiene addirittura che gli fosse stato chiesto da parte del ten. col. Alfano di non farne cenno neppure nel proprio diario, tanto da domandare scherzosamente se dovesse anche «estirpare il ricordo dal cervello». Cfr. AISMO, Fondo Ponzi, b. 156, fasc. 1 “Diari, 1917-1944”, Diario, 5 novembre 1942.

${ }^{46}$ Cfr. GOBETTI, Eric, L'occupazione allegra, cit., pp. 117-120; per i rapporti tra cetnici e le potenze alleate, si veda CUZZI, Marco, op. cit., pp. 42-44.

${ }^{47}$ Sospetti in questa direzione sono presenti in alcuni promemoria del 1943 redatti dal Servizio Informazioni Esercito, cfr. BECHERELLI, Alberto, FORMICONI, Paolo (a cura di), op. cit., p. 112-113.

${ }^{48}$ RODOGNO, Davide, op. cit., p. 252; MONZALI, Luciano, op. cit., pp. 108 et seq.

${ }^{49}$ L'espressione è del generale Quarra Siro, comandante interinale del $6^{\circ} \mathrm{CdA}$, cit. in SALA, Teodoro, «Italiani e cetnici in Jugoslavia (1941-1943). Fonti e linee di ricerca», in Annali della Fondazione Micheletti, 6/1992, pp. 259-269, p. 268.
} 


\section{Alla deriva: l'esperienza della guerra}

Sono felice di sapere che finalmente anch'io potrò fare qualcosa per la Patria in armi, riallacciandomi all'eroico quadriennio 1915-19. Sono ventidue anni e mezzo - dal 16 novembre 1919 - che son borghese. È ora di agire in veste miliare, dopo tanti articoli e tanti discorsi di propaganda. Dio mi aiuterà e mi proteggeràs ${ }^{\circ}$.

La partecipazione al secondo conflitto mondiale rappresentò per Enzo Ponzi un momento di particolare importanza, sia dal punto di vista ideologico che personale. Dopo una quindicina d'anni di marginalizzazione politica, egli vide nella chiamata alle armi la possibilità di riscattarsi di fronte a se stesso e alla Federazione fascista modenese. Pur essendo conscio di non essere più nel pieno delle proprie forze (nel 1942 aveva infatti 48 anni), l'arruolamento fu letto da Ponzi come la naturale ripresa della «marcia interrotta nel 1919-20 [...] verso il compimento del dovere ${ }^{51}$. Molto presto però, il capitano Ponzi entrò in contatto con un mondo ben lontano da quello descritto dalla propaganda fascista in Italia.

In questo senso, i viaggi, una delle tematiche più ricorrenti nei diari di Ponzi, mostrano un paesaggio tutt'altro che confortante, anche grazie alle doti evocative del redattore. Il trasferimento da Modena al comando della Sassari (all'incirca $700 \mathrm{~km}$ ) dura dal 12 al 18 maggio, perché linea ferroviaria tra Oglulin e Knin è stata sabotata in più punti,

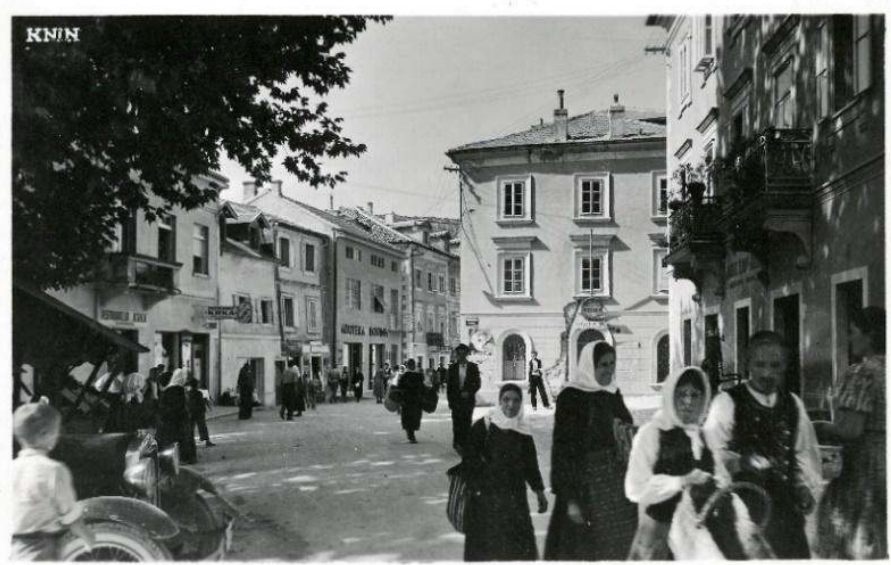

Figura 2: Cartolina ritraente il centro di Knin zona comunque ad alto rischio di

\footnotetext{
${ }^{50}$ AISMO, Fondo Ponzi, b. 156, fasc. 1 "Diari, 1917-1944", Diario, 3 maggio 1942.

${ }^{51}$ AISMO, Fondo Ponzi, b. 156, fasc. 1 "Diari, 1917-1944", Diario, 12 maggio 1942. Si tratta del giorno della partenza per Knin. Poche pagine prima, viene riportato anche lo struggente saluto tra Ponzi e la moglie Elda, con la quale sembra aver avuto momenti anche conflittuali, cosa che contribuì alla scelta di rispondere affermativamente alla designazione della federazione modenese all'ufficio «P» della Sassari. Cfr. AISMO, Fondo Ponzi, b. 156, fasc. 1 "Diari, 1917-1944", Diario, 11 maggio 1942. Ciononostante, la corrispondenza tra i due fu frequente. Le lettere inviate da Ponzi alla moglie, nel corso gli oltre sei mesi coperti dai due diari, sono numerate in ordine progressivo: l'ultima risulta essere la numero centodieci. Cfr. AISMO, Fondo Ponzi, b. 156, fasc. 1 "Diari, 1917-1944", Diario, 18 novembre 1942.
} 
imboscate dei partigiani ${ }^{2}$. Non meno insicuro è il tragitto via nave, perché - racconta Ponzi in diversi punti - sono già molti i convogli a essere stati affondati da sommergibili inglesi nei pressi di Pola, Zara, Šibenik e Spalato53. Giunto finalmente a Knin il 18 maggio, la descrizione del paesaggio e della popolazione è un misto tra quelle fatte dagli esploratori europei in Africa o Asia e quella di uno scenario apocalittico.

Prendo contatto con questo strano pittoresco mondo in cui italiani, croati, serbi, dalmati, mussulmani e bosniaci si incontrano e si accavallano. Straccioni indescrivibili. Facce false e traditrici. Donne schifose che lavorano e sfacchinano. Costumi svariati: dai berrettoni dalmati a forma di fez o di cocuzza [...] agli scialli. [...] Paesaggio quasi sempre orrendo, dirupato, scosceso, brullo, peggio del Carso. Asinelli nani, bestiame piccolo, viti basse: e una massa di bimbi cenciosi e luridi che mendicano. Dappertutto gli italiani stanno sul chi vive, muretti e reticolati di protezione intorno alle stazioni e ai posti di controllo e sorveglianza; le sentinelle tengono impugnato il fucile in posizione di sparo. Appena messo piede a Knin sento la voce tremenda del cannone. Sono 24 anni che le mie orecchie non lo sentivano e ne provo una certa emozione. Che triste arrivo54!

Queste descrizioni, figlie - si potrebbe dire - di una sorta di "orientalismo" diffuso nell'immaginario comune, sono presenti in molti altri casi di memorialistica italiana: i soldati del Regio Esercito erano infatti inseriti in un contesto «coloniale», tra la scarsezza di mezzi, l'abbandono e la paura di un nemico - i partigiani - di cui non si capivano le logiche e le modalità di combattimento55. In alcuni casi li si arrivò perfino ad ammirare, per coraggio e dedizione alla causa ${ }^{56}$.

In generale, in Croazia come nelle altre zone occupate dagli italiani i soldati furono costretti a convivere quotidianamente con l'esperienza della morte e della violenza, sia come vittime che come carnefici, o anche semplicemente come spettatori. Nel caso di Ponzi, sono soprattutto

\footnotetext{
${ }^{52}$ Giunto a Fiume il 13 maggio, Ponzi la descrive come «piena di soldati» e senza un posto dove poter pernottare. In attesa del piroscafo per Zara, è quindi costretto a trasferirsi ad Opatija, utilizzata in quel momento come luogo di ricovero per un gran numero di soldati italiani e tedeschi, per i quali egli mostra una certa ammirazione. Cfr. AISMO, Fondo Ponzi, b. 156, fasc. 1 “Diari, 1917-1944”, Diario, 13 e 14 maggio 1942.

${ }^{53}$ Cfr. AISMO, Fondo Ponzi, b. 156, fasc. 1 “Diari, 1917-1944”, Diario, 17 maggio, 6 e 10 ottobre 1942.

${ }^{54}$ AISMO, Fondo Ponzi, b. 156, fasc. 1 “Diari, 1917-1944”, Diario, 18 maggio 1942. La sottolineatura è nell'originale.

${ }^{55}$ Per considerazioni simili, cfr. SALA, Teodoro, «Guerra e amministrazione in Jugoslavia 1941-1943: un'ipotesi coloniale», in POGGIO, Pier Paolo, MICHELETTI, Bruna (a cura di), Annali della Fondazione Luigi Micheletti : L'Italia in guerra (1940-1943), 5, 1990-1991, pp. 83-94; GOBETTI, Eric, L'occupazione allegra, cit., pp. 180-185.

${ }^{56}$ Ponzi non esterna particolare ammirazione nei confronti dei partigiani, di cui semmai depreca il «fanatismo». Egli riporta infatti due casi di suicidio compiuto da alcuni di essi, facendosi esplodere con una bomba a mano o buttandosi da una finestra, pur di non finire nelle mani delle CC. NN. o dei cetnici. Cfr. AISMO, Fondo Ponzi, b. 156, fasc. 1 “Diari, 1917-1944”, Diario, 7 novembre 1942.
} 
violenze raccontate da altri ufficiali e successivamente viste in fotografia a entrare nel diario, come quelle compiute dai partigiani sui militari italiani, soprattutto ufficiali e camicie nere57. Contrariamente infatti a quanto diffuso dalla propaganda fascista, questi episodi non sembrano essere stati una pratica sistematica contro i soldati semplici: a essere passati per le armi furono principalmente coloro che venivano considerati come i veri responsabili di crimini di guerra ${ }^{5}$. D'altro canto, ciò che manca quasi completamente nel diario di Enzo Ponzi sono i riferimenti alle violenze compiute dagli italiani direttamente contro i prigionieri o contro la popolazione civile, se non in due casi59.

Sebbene non fosse stato coinvolto direttamente in estenuanti cicli operativi, Ponzi si trovò immerso in una realtà di guerra, ben lontana da quella esperita durante il primo conflitto mondiale, più vicina - per certi versi - alle atmosfere cupe di Cuore di tenebra o alla tensione continua e logorante de Il deserto dei tartari.

Nelle pagine finali del secondo diario sulla sua esperienza in Croazia, emergono sempre di più due sensazioni: la disillusione e la frustrazione. Le considerazioni sul ventennale della marcia su Roma, per la quale - dice Ponzi - «tutto diedi, senza nulla chiedere», sono infatti l'occasione per ribadire che, nonostante l'esser stato messo da parte dai colleghi di partito, «nulla [lo avesse]

\footnotetext{
${ }^{57}$ Si tratta di racconti, come quello fattogli durate il viaggio per Knin da un tenente medico modenese in viaggio da Dubrovnik, a tinte forti e carichi di sconcerto: «Evirati, acciecati, segati in due, tagliuzzati come salami a fette, pestatati vivi, sepolti in fosse costruite dai morituri; soprattutto gli ufficiali vengono regolarmente massacrati. Altrettanto le camicie nere. Gli organi genitali mutilati e messi in mano o in bocca alle vittime». Cfr. AISMO, Fondo Ponzi, b. 156, fasc. 1 "Diari, 1917-1944", Diario, 17 maggio 1942. Una volta a Knin, Ponzi troverà la conferma inconfutabile di quanto raccontato: «Non posso più credere che quanto mi raccontano siano esagerazioni: ho visto coi mei occhi fotografie di disgraziati nostri soldati, uccisi, martoriati, sventrati, coi testicoli in mano!», cfr. AISMO, Fondo Ponzi, b. 156, fasc. 1 "Diari, 1917-1944", Diario, 18 maggio 1942. Le immagini della guerra prodotte spontaneamente dai soldati, spesso ritraenti soggetti assenti da quelle ufficiali, venivano fatte circolare privatamente tra commilitoni o inviata a casa ed ebbero una larga diffusione nel corso del conflitto e nel dopoguerra, finendo anche per essere utilizzate come strumento di denuncia dei soprusi, come in PIEMONTESE, Giuseppe, Ventinove mesi di occupazione nella provincia di Lubiana. Considerazioni e documenti, Lubiana, s.e., 1946; cfr. MIGNEMI, Adolfo, La seconda guerra mondiale (1940-1945), Roma, Editori Riuniti, 2000, pp. 15-16 e appendice fotografica; ID., Lo sguardo $e$ l'immagine. La fotografia come documento storico, Torino, Bollati Boringhieri, 2003, pp. 125-132.

${ }^{58}$ Per l'atteggiamento dei partigiani nei confronti dei prigionieri italiani, cfr. riferimenti storiografici, memorialistici e a fonti dell'AUSSME in GOBETTI, L'occupazione allegra, cit. pp 191-192; GIUSTI, Maria Teresa, op. cit., pp. 401-403.

${ }^{59} \mathrm{Nel}$ primo caso si tratta dell'uccisione con una coltellata alla gola di un partigiano fatto prigioniero, mentre nel secondo di un diciottenne arruolato da cetnici, ma «di dubbia fede», ucciso durante la sua fuga da parte di un autiere italiano. Cfr. AISMO, Fondo Ponzi, b. 156, fasc. 1 "Diari, 1917-1944", Diario, 19 maggio e 20 ottobre 1942. Per il vasto tema della repressione e del concentramento in campi dei prigionieri, non trattabile in maniera esaustiva in questa sede, si veda, RODOGNO, Davide, op. cit., pp. 397 et seq.; BURGWYN, H. James, Le tra «professionisti» della controguerriglia italiana in Slovenia e Dalmazia, in BALDISSARA, Luca, PEZZINO, Paolo (a cura di), Crimini e memorie di guerra. Violenze contro le popolazioni e politiche del ricordo, Napoli, L'ancora del Mediterraneo, 2004, pp. 247-259; DI SANTE, Costantino, Italiani senza onore: $i$ crimini in Jugoslavia e i processi negati (1941-1951), Verona, Ombre corte, 2005; FOCARDI, Filippo, Il cattivo tedesco e il bravo italiano. La rimozione delle colpe della seconda guerra mondiale, Roma-Bari, Laterza, 2013; BECHERELLI, Alberto, op. cit., pp. 252-258.
} 
fatto deviare dalla fede nel Duce, dall'amore alla Patria» ${ }^{60}$. Tuttavia, l'emergere tra gli ufficiali della Sassari di atteggiamenti di sfiducia nei confronti del regime, le notizie dei rovesci militari delle truppe dell'Asse in Africa e le contraddizioni presenti nella gestione dei rapporti con le autorità serbe e con quelle croate e, infine, il progressivo ritiro degli italiani anche dai presidi della II zona in Croazia ed Erzegovina contribuirono, al termine del 1942, a incrinare in Ponzi la certezza nella vittoria e a mettere in crisi le grandi aspettative di rivincita anche personale con le quali era partito nella primavera dello stesso anno da Modena ${ }^{61}$.

Te ne accorgi, vecchio Ponzi, che gli anni migliori, gli ultimi della seconda giovinezza se ne vanno? Ti ritroverai, dopo guerra, spostato, spaesato, invecchiato! Ebbene, non importa. Ricomincerò da capo a lavorare, mi rifarò la clientela, e dagli acciacchi e vecchiezza troverò conforto nelle mie amate collezioni. Certo non vorrò più scannarmi e sfacchinare come in passato $^{62}$.

Confrontando queste parole, scritte a sei mesi dalla chiamata alle armi, con quelle dell'inizio del diario si nota come la prospettiva sia profondamente cambiata e come l'esperienza del conflitto abbia portato l'avvocato di Modena a rivedere molte delle premesse ideologiche, senz'altro legate anche a volontà di affermazione personale, con le quali era partito. Non da ultimo, questo commento rispecchia per certi versi il meccanismo di "rimozione" dalla memoria collettiva a cui andò incontro l'Italia nell'immediato dopoguerra, quando cioè si evitò di fare i conti col proprio operato come potenza occupante tra il 1940 e il 1943, preferendo elaborare una narrazione da "nazione-vittima".

\footnotetext{
${ }^{60}$ AISMO, Fondo Ponzi, b. 156, fasc. 1 “Diari, 1917-1944”, Diario, 17 ottobre 1942.

${ }^{61}$ Era infatti andata in fumo anche la speranza di essere nominato federale della Federazione fascista modenese, per la qual cosa Ponzi aveva tenuto fitti contatti epistolari con amici e colleghi di partito a casa. Cfr. AISMO, Fondo Ponzi, b. 156, fasc. 1 "Diari, 1917-1944”, Diario, 17 e 21 ottobre 1942.

${ }^{62}$ AISMO, Fondo Ponzi, b. 156, fasc. 1 “Diari, 1917-1944”, Diario, 11 novembre 1942.
} 


\section{L'AUTORE}

Francesco MANTOVANI (Modena, 1991) ha portato a termine nel luglio 2016 il percorso di Laurea magistrale nell'ambito del corso integrato italo-tedesco organizzato dall'Università di Bologna e da quella di Bielefeld, discutendo una tesi intitolata Le associazioni imprenditoriali in Italia e Germania durante la Grande guerra. Attualmente iscritto al Master di $2^{\circ}$ livello in Public History presso l'Università di Modena e Reggio Emilia, si interessa del primo Novecento e di comunicazione storica.

URL: < http://www.studistorici.com/progett/autori/\#Mantovani > 